\title{
Sex hormones impact vascular calcification and fracture in dialysis patients
}

\author{
Won Suk An \\ Department of Internal Medicine, Dong-A University College of Medicine, Busan, Republic of Korea
}

Fracture events are closely related to morbidity and mortality, especially in dialysis patients. The incidence of fractures was reported to be 1.8 to eight times higher in dialysis patients compared to the regional general population [1]. Dialysis patients with fracture showed a fourfold higher mortality and hospitalization rate compared to patients without fracture. Fractures are definitely related to osteoporosis in postmenopausal women and are also common in women undergoing chronic hemodialysis (HD). Older age, female sex, and low serum albumin concentration were all predictors for new fracture risk in the Dialysis Outcomes and Practice Patterns Study (DOPPS, 2002-2004) [2].

Vascular calcification (VC) is a good predictor for cardiovascular disease, morbidity, and mortality. VC is positively associated with vertebral fractures and is inversely correlated with bone mineral density (BMD) in populations without chronic kidney disease [3]. Lower serum fetuin-A level and presence of VC on thoracic and lumbar lateral views were independently linked to a higher risk of incident fracture in a Taiwanese prospective, observational cohort study of 685 dialysis patients [4]. Higher VC score was independently associated with increased risk

Received July 18, 2020; Accepted July 21, 2020

Editor: Tae-Hyun Yoo, Yonsei University, Seoul, Republic of Korea Correspondence: Won Suk An

Department of Internal Medicine, Dong-A University College of Medicine, 32 Daesingongwon-ro, Seo-gu, Busan 49201, Republic of Korea.E-mail:anws@dau.ac.kr

Copyright (@ 2020 by The Korean Society of Nephrology

(a) This is an open-access article distributed under the terms of the Creative Commons Attribution Non-Commercial License (http://creativecommons. org/licenses/by-nc-nd/4.0/), which permits unrestricted non-commercial use, distribution, and reproduction in any medium, provided the original work is properly cited. of fracture in $341 \mathrm{HD}$ patients. Elderly and female dialysis patients also had higher fracture risk based on a retrospective cohort study [5]. Therefore, VC is an important predictor of possible fracture, especially in postmenopausal women on dialysis.

Estrogen deficiency after menopause induces a decrease in BMD by stimulating differentiation of osteoclast precursors and activating mature osteoclasts. Osteoporosis related to lower serum estradiol level is associated with high risk of fracture. Estrogen can prevent VC by inhibiting receptor activator of nuclear factor kappa B ligand (RANKL) signaling pathways. Estrogen replacement reduced bone morphogenetic protein-2, which is a calcification inducer, and increased the matrix gla protein, which is a calcification inhibitor, in both in vitro and in vivo studies. Endogenous estrogens consist of estrone, 17 $\beta$-estradiol (E2), and estriol. E2 is the main and active form and works via estrogen receptor-alpha (ER$\alpha$ ) and -beta. Growth arrest-specific gene 6, a member of the vitamin K-dependent protein family, is a regulatory target of E2/ER- $\alpha$ signaling in vascular smooth muscle cell calcification. Long-term E2 supplementation was associated with lower coronary artery calcium score in postmenopausal women aged 50 to 59 years compared to those who received placebo. Estradiol concentration is usually low in menopausal women treated with dialysis compared to the general population. Therefore, it is suspected that postmenopausal dialysis patients have high risk of VC and fracture.

In this issue of Kidney Research and Clinical Practice, Nam et al [6] showed that the aortic calcification index (ACI) on abdominal computed tomography was independently associated with incident fracture in 593 dialysis patients (male, $n=328$; female, $n=265$ ). The fracture 
incidence rate was $12 \%$ during a median follow-up period of 36.7 months, and fractures of the lower extremities were more frequent than vertebral fractures $(62.4 \%$ vs. $14.0 \%$ ) in this retrospective study including 169 peritoneal dialysis patients. It is noteworthy that ACI can be a reliable predictor of future fracture in female patients with end-stage renal disease but not in male patients. Postmenopausal females between 60 to 79 years of age had a higher incidence of fracture events compared to males (30 fractures vs. 16 fractures), although there are no data on BMD, sex hormones, and axial skeletal muscle mass. This difference of fracture events and sex-specific differences distinctly appeared after the five-year follow-up. Therefore, this study may support preventive management for osteoporosis, which is necessary especially in postmenopausal dialysis patients with higher ACI.

Estrogen is considered one of the most effective drugs for osteoporosis treatment in postmenopausal women. However, potential adverse effects of estrogen on the breast and endometrium should be considered, and thromboembolism can be an associated complication of estrogen use, especially in HD patients with vascular access thrombosis risk. Transdermal E2 and noretisterone acetate application over 12 months showed BMD improvement, but two recurrent vascular access thromboses were reported in 10 pre-menopausal women receiving HD [7]. Selective estrogen receptor modulators, such as raloxifene at a dose of $60 \mathrm{mg} /$ day, improved trabecular $\mathrm{BMD}$, reduced serum calcium level, and increased serum intact parathyroid hormone level in 17 postmenopausal women on HD [8]. There were no cases of breast cancer or clotting problems due to vascular access or thromboembolism in several small studies using raloxifene $[8,9]$. Therefore, long-term studies are needed to confirm the safety and beneficial effect of raloxifene for fracture prevention in post-menopausal women undergoing dialysis treatment.

Testosterone, which impacts muscle mass, may reduce VC by reducing RANKL signaling and suppressing of local inflammatory signaling. A recent study [6] showed that the median ACI was significantly higher in female dialysis patients than male dialysis patients. Abdominal aortic calcification on plain radiograph was more prevalent and severe in elderly Australians (female $62 \%$ ) with low sex-specific, body mass index-normalized appendicular muscle mass [10]. Muscle mass and strength are almost
$50 \%$ lower in young adult women compared to men, and the risk of falls and fractures is related to muscle mass/ strength. Therefore, male dialysis patients with relatively higher muscle mass and adequate testosterone level may experience fewer VCs and fractures. However, further prospective controlled trials are necessary to elucidate the roles of testosterone and muscle mass on $\mathrm{VC}$ and fracture in males because there was no significant interaction between the high ACI group, log ACI, and incident fracture in male dialysis patients in this study.

Androgens and estrogens are synthesized from cholesterol. Therefore, very low cholesterol levels caused by malnutrition may reduce sex hormone production in dialysis patients. Testosterone is converted to estrogen by the aromatase enzyme (CYP19A1) in both females and males. Circulating testosterone and estradiol are tightly bound to the liver-derived glycoprotein sex hormone-binding globulin (SHBG). Disruption to gonadal hormones is frequent, as observed in $50 \%$ to $75 \%$, in men undergoing dialysis. Higher SHBG level is significantly associated with non-vertebral fracture in young male dialysis patients without association of testosterone or E2 level. Free testosterone and E2 concentrations, which are more involved with maintaining bone health rather than total testosterone and $\mathrm{E} 2$ levels, may be related to the as-

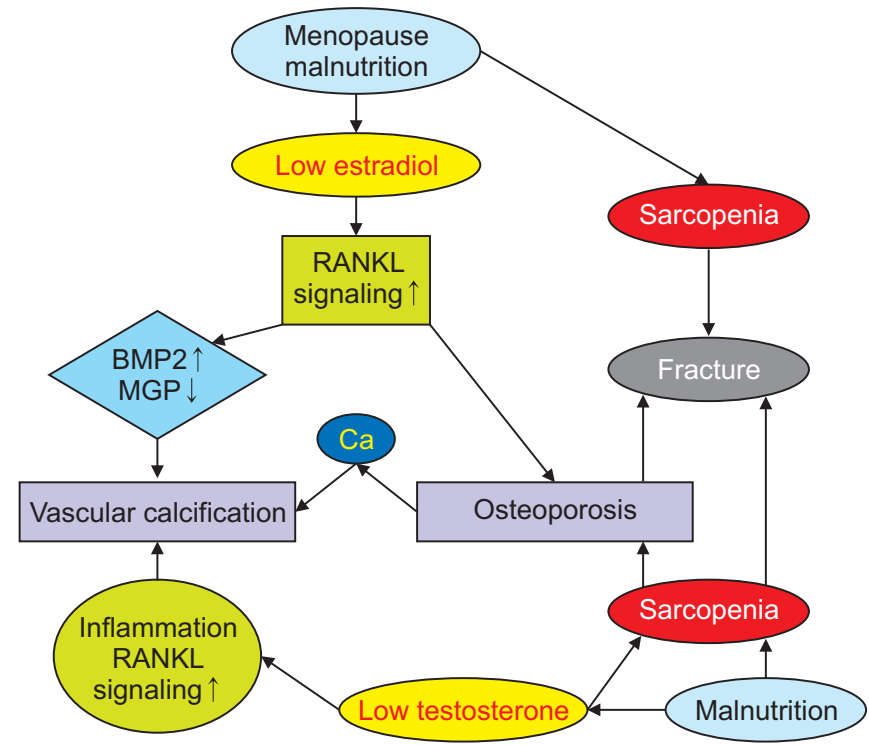

Figure 1. Possible mechanism for vascular calcification, osteoporosis, and fracture caused by decreased sex hormones. BMP2, bone morphogenetic protein 2; Ca, calcium; MGP, matrix Gla protein; RANKL, receptor activator of nuclear factor kappa B ligand. 
sociation of SHBG with fracture, although this relationship is unclear. Therefore, additional studies are necessary to determine the role of sex hormones and related proteins in fractures in male and female dialysis patients.

The calcification paradox, which involves increased arterial mineralization accompanied by decreased BMD, is frequently found in dialysis patients with osteoporosis and VC. Decreased sex hormone levels, which have a causal relationship with malnutrition and sarcopenia, may be associated with VC, osteoporosis, and fracture in dialysis patients (Fig. 1). Based on the current study, a sex-specific relationship between VC and incident fracture and sex hormones may be important for managing and preventing $\mathrm{VC}$ and fracture.

\section{Conflicts of interest}

The author has no conflicts of interest to declare.

\section{Funding}

This study was supported by the Dong-A University research fund.

\section{References}

[1] Tentori F, McCullough K, Kilpatrick RD, et al. High rates of death and hospitalization follow bone fracture among hemodialysis patients. Kidney Int 2014;85:166-173.

[2] Jadoul M, Albert JM, Akiba T, et al. Incidence and risk factors for hip or other bone fractures among hemodialysis patients in the Dialysis Outcomes and Practice Patterns
Study. Kidney Int 2006;70:1358-1366.

[3] Naves M, Rodríguez-García M, Díaz-López JB, GómezAlonso C, Cannata-Andía JB. Progression of vascular calcifications is associated with greater bone loss and increased bone fractures. Osteoporos Int 2008;19:1161-1166.

[4] Chen HY, Chiu YL, Hsu SP, Pai MF, Yang JY, Peng YS. Relationship between fetuin A, vascular calcification and fracture risk in dialysis patients. PLoS One 2016;11:e0158789.

[5] Matias PJ, Laranjinha I, Azevedo A, et al. Bone fracture risk factors in prevalent hemodialysis patients. J Bone Miner Metab 2020;38:205-212.

[6] Nam YJ, Hwang SY, Kim DW, Kim D, Shin SJ, Yoon HE. Sexspecific relationship between vascular calcification and incident fracture in patients with end-stage renal disease. Kidney Res Clin Pract 2020;39:344-355.

[7] Matuszkiewicz-Rowiñska J, Skórzewska K, Radowicki S, et al. The benefits of hormone replacement therapy in premenopausal women with oestrogen deficiency on haemodialysis. Nephrol Dial Transplant 1999;14:1238-1243.

[8] Tanaka M, Itoh K, Matsushita K, et al. Effects of raloxifene on bone mineral metabolism in postmenopausal Japanese women on hemodialysis. Ther Apher Dial 2011;15 Suppl 1: 62-66.

[9] Saito O, Saito T, Asakura S, et al. The effects of raloxifene on bone turnover markers and bone mineral density in women on maintenance hemodialysis. Clin Exp Nephrol 2011; 15:126-131.

[10] Hernández E, Valera R, Alonzo E, et al. Effects of raloxifene on bone metabolism and serum lipids in postmenopausal women on chronic hemodialysis. Kidney Int 2003;63:22692274 . 